\title{
Gene-expression Analysis Identifies Specific Patterns of Dysregulated Molecular Pathways and Genetic Subgroups of Human Hepatocellular Carcinoma
}

\author{
HOLGER G. HASS ${ }^{1}$, ULRICH VOGEL ${ }^{2}$, MICHAEL SCHEURLEN ${ }^{3}$ and JÜRGEN JOBST ${ }^{4}$ \\ ${ }^{1}$ Department of Oncology, Paracelsus Hospital, Scheidegg, Germany; \\ ${ }^{2}$ Department of Pathology, University of Tuebingen, Tuebingen, Germany; \\ ${ }^{3}$ Department of Gastroenterology, Hematology, Oncology and Rheumatology, \\ University of Wuerzburg, Wuerzburg, Germany; \\ ${ }^{4}$ Matrigene GmbH, Reutlingen, Germany
}

\begin{abstract}
Background. Hepatocellular carcinoma comprises of a group of heterogeneous tumors of different etiologies. The multistep process of liver carcinogenesis involves various genetic and phenotypic alterations. The molecular pathways and driver mutations involved are still under investigation. Materials and Methods: DNA micorarray technology was used to identify differentially expressed genes between human hepatocarcinoma and non-tumorous liver tissues to establish a unique specific gene-expression profile independent of the underlying liver disease. The validity of this global gene-expression profile was tested for its robustness against biopsies from other liver entities (cirrhotic and non-cirrhotic liver) by diagnosing HCC in blinded samples. Results: Most of the consistently and strongly overexpressed genes were related to cell-cycle regulation and DNA replication [27 genes, e.g. cyclin B1, karyopherin alpha 2 (KPNA2), cyclin-dependent kinase 2 (CDC2)], G-protein depending signaling [e.g. Rac GTPase activating protein 1 (RACGAP1), Rab GTPase YPT1 homolog (RAB1), and ADPribosylation factor-like 2 (ARL2)] and extracellular matrix remodelling or cytoskeleton structure [22 genes, e.g. serine proteinase inhibitor 1 kazal-type (SPINK1), osteopontin (OPN), secreted protein acidic and rich in cysteine (SPARC), collagen type 1 alpha2 (COL1A2), integrin alpha6 (ITGA6), and
\end{abstract}

This article is freely accessible online.

Correspondence to: Holger G. Hass, Department of Oncology, Paracelsus Hospital, Kurstrasse 5, 88175 Scheidegg, Germany. Tel: +498381501225, Fax: +49 8381501290,e-mail: dr.holger.hass@ paracelsus-kliniken.de

Key Words: Hepatocellular carcinoma, gene expression analysis, SPINK1, glypican 3, hepatocarcinogenesis, oligonucleotide arrays, affymetrix. metalloproteinase 12 (MMP12)]. Furthermore, significantly differentially expressed genes (e.g. calcium-binding proteins, $G$ proteins, oncofetal proteins) in relation to tumor differentiation were detected using gene-expression analysis. Conclusion: It is suggested that these significantly dysregulated genes are highly specific and potentially utilizable as prognostic markers and may lead to a better understanding of human hepatocarcinogenesis.

Hepatocellular carcinoma (HCC) is the fourth most common tumor type on a global basis today. In Asia and Africa, HCC is one of the major causes of cancer death due to its high frequency and poor prognosis (1). Well-known risk factors for acquiring HCC include chronic infection with hepatitis $\mathrm{B}(\mathrm{HBV})$ or $\mathrm{C}(\mathrm{HCV})$ virus, increasing incidence of obesity leading to liver inflammation, and prolonged dietary consumption of aflatoxins. More recently, it has been shown that the relative risk for the development of HCC is increased synergistically among individuals exposed to both aflatoxins and $\mathrm{HBV}(2,3)$ and among $\mathrm{HCV}$-positive individuals with increased alcohol consumption (4). The incidence of $\mathrm{HCC}$ is expected to increase significantly in the next decade, mainly due to the increasing number of patients infected with chronic $\mathrm{HCV}$ and non-alcoholic steatohepatitis (NASH) especially in Western countries. Despite the different etiological factors and underlying liver diseases HCC is a heterogeneous cancer. Continuous inflammation occasionally damages DNA in the hepatocytes of the regenerating liver, thereby increasing the chances of gene alteration related to carcinogenesis $(5,6)$. Recent advances in molecular genetics have identified various genetic abnormalities related to hepatocarcinogenesis, which are helpful in elucidating the pathogenesis and development of HCC. Gross abnormalities of karyotypes or chromosomal subregions, such as loss of heterozygosity ( $\mathrm{LOH}$ ) or loss of some chromosomal parts, have been found in a significant 
proportion of HCC. An extended study to analyze the allelotype in HCC and identify candidate sites for promoter genes in the development of HCC has indicated allelic losses in regions of tumor-suppressor genes on different chromosomes $(5 \mathrm{q}, 10 \mathrm{q}, 11 \mathrm{p}, 16 \mathrm{q}, 17 \mathrm{p})$ and especially in chromosome 8 (7-9). Altered gene expression due to either mutations or changes in its regulation characteristics in HCC compared to corresponding non-tumor tissues were also typically observed in human hepatocarcinogenesis. The integrity of various cell cycle-related oncogenes such as cellular myelocytomatosis oncogene $(c-M Y C)(10)$, Kirsten rat sarcoma viral oncogene homolog $(K-R A S)$, Neuroblastoma RAS viral oncogene homolog $(N-R A S)(11$, 12) and tumor-suppressor genes such as retinoblastoma protein $(R B)(13)$ and p53 (3) are known to undergo genetic changes. Despite these interesting observations, the multistep process and major driver pathways of human carcinogenesis are still unclear. This fact and the increasing worldwide incidence of HCC underline the importance and need for molecular genomic research into this type of human cancer.

In this study, we used oligonucleotide microarrays to perform a large-scale expression analysis to identify specific altered molecular pathways and candidate target genes in human hepatocarcinogenesis. In the last decade, the identification of genes in different types of human cancer has already been performed using this technology (14-18). In addition, HCC has already been analyzed by microarray techniques (19-22). Most of these studies have been able to identify further genes putatively involved in hepatocarcinogenesis, but in most cases, specific subgroups of HCC (especially HBV-, HCV- or aflatoxine B-induced HCC) were analyzed.

In the present study, we present a unique $\mathrm{HCC}$-specific gene-expression profile based on a comprehensive microarray study with HCC samples involving etiologies such as hepatitis B and C, as well as other common causes such as alcoholic liver disease or NASH in an European population. This gene-expression profile was tested for its robustness by differentiating it not only against cirrhotic and normal liver tissue but also other malignant liver lesions such as cholangiocellular carcinoma (CCC). Furthermore, specific dysregulated molecular pathways and genes in relation to tumor differentiation and evolution (grading) were described for the first time here in human hepatocarcinogenesis.

\section{Materials and Methods}

Patients and ethics. Primary HCCs and their surrounding noncancerous liver tissue were obtained from patients undergoing surgical resection of primary HCC. Surgical specimens were obtained from 33 patients with HCC (33 tumors, together with 20 corresponding non-malignant liver tissue samples), and control liver tissue from 18 other patients (eight biopsies of non-diseased liver; 10 biopsies of patients with intrahepatic CCC). Patients underwent surgical resection according to standard procedures. No patient received preoperative chemoembolization or systemic therapy (e.g. sorafenib; for more details see Table I). In accordance with the Declaration of Helsinki, Ethics Committee approval was obtained (no.194/2001V). Parts of resected samples were only used for further genomic analysis after written informed consent was obtained from the patients.

Samples of sufficient weight $(>400 \mathrm{mg}$ ) from malignant and corresponding non-malignant liver tissue were excised and snapfrozen in liquid nitrogen within $20 \mathrm{~min}$ after excision and shipped with a pathology report, a clinical summary and the result of virological testing, under appropriate conditions to maintain specimen integrity. Until further sample processing, the biopsies were stored at $-80^{\circ} \mathrm{C}$.

Histopathological evaluation. Pathological reports with tumor typing, staging (performed using Union for International Cancer Control criteria) and grading as well as clinical data were obtained for each tissue sample. Hematoxylin-eosin staining was performed for detection of features such as bile canalicular structure and Mallory hyaline bodies. Additional histochemical and immunohistochemical stainings with a panel of antibodies were used routinely [anti-hepatocyte clone OCH1E5 (HEP-PAR-1), alphafetoprotein (AFP), cytokeratin 7 (CK7), carbohydrate antigen 19-9 (CA19-9), cluster of differentiation 10 (CD10), carcinoembryonic antigen (CEA)] for all resected tissue biopsies to confirm the histological diagnosis of HCC and to exclude other types of malignancy.

Preparation of labeled cRNA and hybridization to oligonucleotide arrays. For transcription of the cleaned total RNA into doublestranded cDNA, the SuperScript Choice system (Invitrogen, Carlsbad, CA, USA) was used. First-strand cDNA synthesis was primed with a T7-(dT24) oligonucleotide primer with a RNA polymerase promoter site added to the 3 'end. After second-strand synthesis, in vitro transcription was performed in the presence of biotin-11-cytidine triphosphate (CPT) and biotin-16-uridine triphosphate (UTP) (Enzo Diagnostics, New York, NY, USA) to produce biotin-labelled cRNA. cRNA products were fragmented $\left(20 \mu \mathrm{g}\right.$ at $94^{\circ} \mathrm{C}$ for $\left.35 \mathrm{~min}\right)$ in 35 200 bases in length and added to a hybridization solution to a final cRNA concentration of $0.05 \mathrm{mg} / \mathrm{ml}$. Hybridization was performed by incubation (18-20 h) of $200 \mu \mathrm{l}$ of the sample with an Affymetrix human GeneChip (Hu133A) containing 22,283 probe sets for known genes or expressed sequence tags (ESTs) and stained with streptavidin-phycoerythrin. A Gene Array scanner G2500A (Hewlett Packard, Palo Alto, CA, USA) was used for scanning according to the procedures developed by Affymetrix.

Data mining, statistical analysis, tumor sub-classification and identification of significantly altered metabolic pathways involved in human hepatocarcinogenesis. The statistical analyses and presentation of the obtained data were performed in accordance with MIAME criteria and will be published at (http://www.paracelsuskliniken.de/scheidegg/fuer-fachkreise/forschung/gene-profiles).

Raw data analysis was conducted using the Affymetrix microarray suite (MAS vs5.0.1). MAS produced an expression value plus an index parameter indicating positive or negative detection (present call index) for each of the 22,283 probe sets (known genes/ESTs) on the array. Statistical analysis and post-processing were performed using GeneSpring (vs 6.1; Silicon Genetics, 
Table I. Clinical and histopathological findings of the study population $(n=33)$.

\begin{tabular}{lc}
\hline Characteristic & $\mathrm{n}(\%)$ \\
\hline Mean age (years) & $60.7(10.8)$ \\
Gender (male) & $19(57.5)$ \\
Tumor etiology & $4(12.1)$ \\
HBV & $10(30.3)$ \\
HCV & $10(30.3)$ \\
C2 & $4(12.1)$ \\
NASH & $2(6.0)$ \\
Metabolic (hemochromatosis, alpha1-AT deficiency) & $3(9.1)$ \\
N.S. & \\
Tumor stage (TNM) & $3(9.0)$ \\
T1 & $11(33.3)$ \\
T2 & $14(42.4)$ \\
T3 & $5(15.1)$ \\
T4 & \\
Tumor grading & $2(6.0)$ \\
G1 & $17(51.6)$ \\
G2 & $14(42.4)$ \\
G3 & \\
\hline
\end{tabular}

HBV: Chronic hepatitis B virus; $\mathrm{HCV}$ : chronic hepatitis $\mathrm{C}$ virus; $\mathrm{C} 2$ : alcohol abuse; NASH: non-alcoholic steatohepatitis; N.S.: not specified.

Redwood City, CA, USA) and GeneExplore (vs1.1; AppliedMaths, Sint-Martens-Laten, Belgium) software. Mismatch probes acted as specific controls on each array and allowed the direct subtraction of both background and cross-hybridization signals. Only Chip results of different scaling factors of 0.5 to 1.8 were accepted for further analyses. Expression values were then $\log _{2}$-transformed on the basis of the signal $\log$ ratio which is given by the comparison of twoarray results between tumorous and non-tumorous tissues.

A $p$-value of less than 0.05 ( $t$-test) and a fold-change of $\geq 2$ in $60 \%$ or more of all analyzed samples were considered as significant

For the detection of statistically differentially expressed genes in well- and poorly differentiated tumors (genetic subclassification by tumor grading) a one-way ANOVA test was performed. In a second step, significantly dysregulated genes $(p<0.05)$ of the tumor samples were used for a weighted two-dimensional clustering.

For identifying specific signaling or metabolic pathways in human hepatocarcinogenesis, the data of significantly differentially expressed genes were transferred to a public domain software program (GenMAPP 2.0 beta $^{\circledR}$; Gladstone Institutes, San Francisco, CA, USA). This software allows for the graphic depiction of various pathways and using the expression levels of the involved genes shows up- or down-regulation of the specific pathway.

Validation of expression data by real-time polymerase chain reaction (RT-PCR; LightCycler ${ }^{\mathcal{O}}$ System). For PCR analysis, four genes [karyopherin alpha 2 (KPNA2), glypican 3 (GPC3), serine protease inhibitor type kazal 1 (SPINK1), and squalene monooxygenase (ERG1)] found to be significantly overexpressed in at least $80 \%$ of the HCC tissue samples as determined by the microarray analysis were chosen and primers corresponding to the coding regions determined were used for RT-PCR analyses using the LightCycler ${ }^{\odot}$ system (Roche Diagnostics, Mannheim, Germany).
Gene-specific primers corresponding to the coding region were designed using OLIGO primer analysis software (Molecular Biology Insights, Colorado Springs, CO, USA) and were obtained from Biomers.net (Ulm, Germany). Primer sequences were as follows: SPINK1: 151U: GCCTTGGCCCTGTTGAGTCTA, 1:273L: CACG CATTCATTGGGATAAGTATTT; GPC3: 1808U: CAGCAGGCAAC TCCGAAGG, 1929L: TGGGCACCAGGCAGTCAGT; KPNA2: 328U: GAAAACCGCAACAACCA, 501L: GCCCAAGAAGG ACACAAAT; ERG1: 1640U: CAAACTTGGTGGCGAATG, 1738L: AAGCAAAAATACACGGCATAGA.

Preliminary experiments were carried out to test for specificity of product formation, determine annealing temperatures, and check for $\Delta \mathrm{T}_{\mathrm{m}}$ (product-primer dimer) $>3^{\circ} \mathrm{C}$. Validation experiments were performed within a fluorescent signal window lacking primer-dimer formation. The correct PCR efficiency for each target was determined by constructing relative standard curves using five-point halflogarithmic RNA dilutions from one sample. RT-PCR reactions were performed using LC RNA Amplification Kit SYBR Green I, (Roche Diagnostics). Amplification was followed by melting-curve analysis. Relative values for the initial target concentration in each sample were determined using LightCycler software 3.5. The relative change in gene expression was computed by pairwise comparison of tumor samples to samples of adjacent normal tissue for each patient.

\section{Results}

Gene-expression profiling of HCC. In the present study we evaluated 33 HCC samples by oligonucleotide microarray analysis, comparing each sample with a corresponding nonmalignant sample from the adjacent tissue.

All primary Chip data were screened for RNA quality by 5'-3' degradation. Of 22,283 probe sets present on the chip, on average $42.6 \%$ of genes in HCC and $39.8 \%$ in normal samples (difference not significant) were expressed in the liver tissue samples.

Another aim of the present study was to identify a unique pattern of gene expression for HCC that could be used for diagnosis in comparison to other primary or secondary liver tumors. To accomplish this, a hierarchical cluster analysis was performed using all of the $\sim 13,000$ expressed genes in all analyzed tissue probes. Using this method, a cluster was produced which showed consistent up- and down-regulated genes independently of the primary cause of the liver disease $\left(\mathrm{HCV}^{-} v s . \mathrm{HBV}^{-} v s\right.$. alcoholic-induced $\left.\mathrm{HCC}\right)$ and gender of the patients.

Based on the primary data, an algorithm was developed to identify and rank the most consistently up- and down-regulated genes. All genes at least 2-fold overexpressed in at least $60 \%$ of the HCC tumor samples and all genes whose expression was reduced by at least $50 \%$ in at least $60 \%$ of the tumor samples were considered to be significantly differentially expressed in HCC versus non-malignant liver tissue. Using this method, a databank of 1,085 genes was generated.

For further refinement, the restricted list of 1,085 genes was finally used for clustering analysis. This resulted in a far more homogenous cluster, which was able to differentiate 
clearly all HCC tumor samples from all other liver tissue samples (corresponding non-malignant liver tissue; see Figure 1) and even other, malignant liver tumors.

To further test the robustness of this cluster, a supervised learning testing based on neuronal networking was applied. For this approach, all $33 \mathrm{HCCs}$ and 20 corresponding nonmalignant probes were used. For the initial training and testing, all probes were used. This resulted in a positive predictive value for $\mathrm{HCC}$ of $92 \%$ and a negative predictive value of $100 \%$ of the gene cluster with the expression pattern of 350 genes. With the additional information of the geneexpression pattern of the remaining genes (of the 1,085 ) this gives striking security in obtaining a diagnosis of HCC in comparison to standard histopathology.

Dysregulated genes and altered signaling and metabolic pathways in hepatocarcinogenesis. Of these 1,085 significantly dysregulated genes/ESTs, 450 were upregulated and 635 down-regulated. To evaluate the involved pathways in human hepatocarcinogenesis, the 1,085 significantly dysregulated genes were transferred in the GeneMAPP ${ }^{\circledR}$ program and checked with gene descriptions in gene databases such as UniGene ${ }^{\circledR}$ (U.S. National Library of Medicine, Bethesda, MD, USA) and GeneCards ${ }^{\circledR}$ (Weizmann Institute, Rehovot, Israel). Using this approach, out of the 1085 genes/ESTs, 648 genes were allocated to specific metabolic and signaling pathways.

Most of the consistently and strongly overexpressed genes were related to cell-cycle regulation and DNA replication [27 genes, e.g. cyclin B1 (CCNB1), KPNA2, tubulin gamma 1 (TUBG1), cell division control 25 beta and $46(C D C 25 B$, $C D C 46)$, ribonucleoside-diphosphate reductase M2 (RRM2), topoisomerase II alpha (TOP2A), calcyclin (S100AO), proliferating cell nuclear antigen (PCNA); see Figure 2) or Gprotein depending signaling [seven genes, e.g. Rho family GTPase 3 (RND3), Ras homolog enriched in brain (RHEB), Rac GTPase activating protein 1 (RACGAP1), ADP-ribosylation factor-like $2(A R L 2)$ and other members of the RAS oncogene family $(R A N, R A B 16)]$. Most of the other up-regulated genes attributed to gene families such as those coding for extracellular matrix, cell adhesion molecules and cytoskeleton structure [22 genes, e.g. SPINK1, osteopontin $(O P N)$, secreted protein acidic and rich in cysteine (SPARC), different collagens as collagen type 1 alpha2 (COL1A2), collagen type 4 alpha2 (COLAA2) and collagen type 6 alpha3 (COL6A3), tropomyosin 2 beta (TPM2), capping protein $(G A P G)$, integrin alpha6 (ITGA6) and metalloproteinase 12 (MMP12)].

In line with up-regulation of genes involved in cell-cycle regulation and DNA replication, a variety of genes coding for ribosomal protein synthesis [seven genes, e.g. 60S ribosomal protein L39 (RPL39L), ribosomal protein 7 (RPS7) and ribosomal protein SA (RPSA)] and proteasome degradation $[12$ genes, e.g. $26 \mathrm{~S}$ proteasome regulatory subunit $\mathrm{S} 1$ and 2 (PSMD1, PSMD2) and ubiquitin-like protein $5(U B L)$ ] were also up-regulated.

In contrast, pro-apoptotic genes as DNA fragmentation factor subunit beta $(D F F B)$ and nuclear factor kappa B subunit 1 (NFKB1a) were down-regulated, whereas apoptosis-inhibitory genes as kinetochore protein fta7 $(C N L 3)$ and chromosome segregation 1-like (CSE1L) were overexpressed in tumor tissue.

Proteins involved in specific metabolic pathways such as aminoacid metabolism (13 genes), carbohydrate and fat metabolism (10 and eight genes) or detoxification and electron transport (18 genes) were also down-regulated compared with the expression profile of the non-malignant, corresponding liver tissue.

Interestingly, especially in virally induced HCC, genes coding for interferon-stimulated proteins and interleukins such as guanylate-binding protein 2 (GBP2), ISG15 ubiquitin-like modifier (ISGI5) and interferon-alpha inducible protein 27 (IFI27) were overexpressed in comparison to the corresponding, in most cases, cirrhotic liver tissue.

For the sake of clarity, only the most consistently overexpressed (fold change $\geq 2$ in $\geq 80 \% ; p<0.0001$ ) genes were categorized and are listed in Table II.

Detection of specific altered gene-expression levels in correlation with tumor differentiation. For sub-classification of HCC in relation to histopathological differentiation (grading), a weighted two-dimensional clustering of tumor samples was performed. All expressed genes/ESTs in tumor samples (42.6\% of all genes/ESTs of the array) were grouped according to histological grading of the sample, ranging from 1 to 3 , and then ranked according to their differential expression values. Differentiation of the gene-expression profiles of the tumor samples according to histopathological grading of 1 and 3 identified a total of 186 dysregulated genes $(p<0.05$ and two-fold up- or down-regulation in $60 \%$ or more), which were used for a weighted two-dimensional cluster analysis and a conditional gene-tree analysis based on one-way ANOVA. Despite the heterogeneity and different etiology of the analyzed tumor probes and the small group of G1 tumors $(n=2)$, it was not possible to establish a significant gene-expression profile. Interestingly, a significant upregulation of genes involved in calcium signaling such as S100 calcium-binding protein A8, A9, A11 and P (S100A8, S100A9, S100A11, S100P) and neurotensin (NTS), and for specific $\mathrm{G}$ antigens as $G A G E 2,-3,-4,-6$ and -7 were detected in poorly differentiated tumor samples (G3) when compared to well- and moderately differentiated tumor samples (G1, G2). Genes coding for fetal liver proteins such as alphafetoprotein (AFP) and neurotensin were also significantly overexpressed $(p<0.0001)$ in poorly differentiated tumors. The 20 most dysregulated genes in G3 tumors are listed in Table III. 


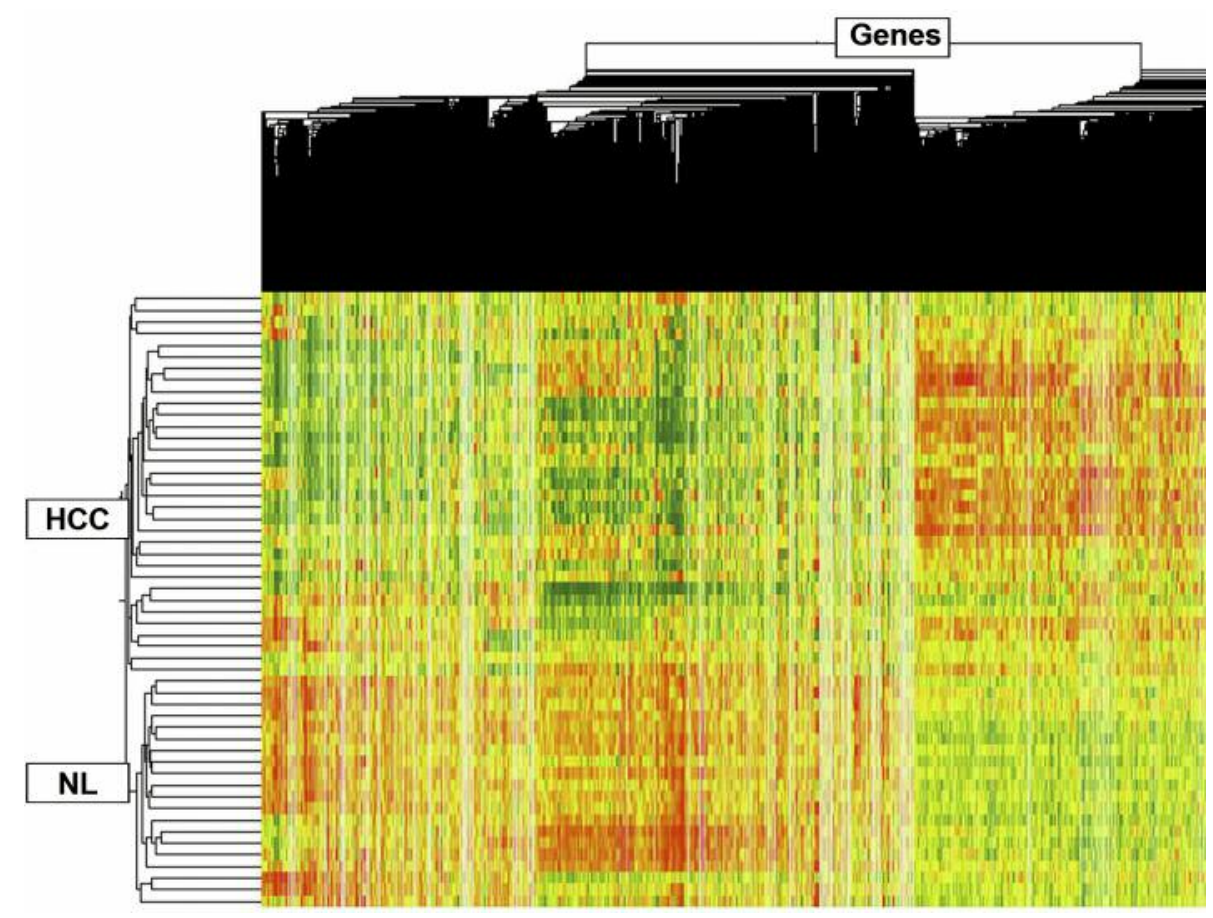

Figure 1. Two-dimensional cluster analysis using 1,085 dysregulated genes in hepatocellular carcinoma vs. non-malignant corresponding liver tissue (NL) (2-fold change in $\geq 60 \%$ ). Red: Up-regulated genes; green: down-regulated genes; $p<0.05$.

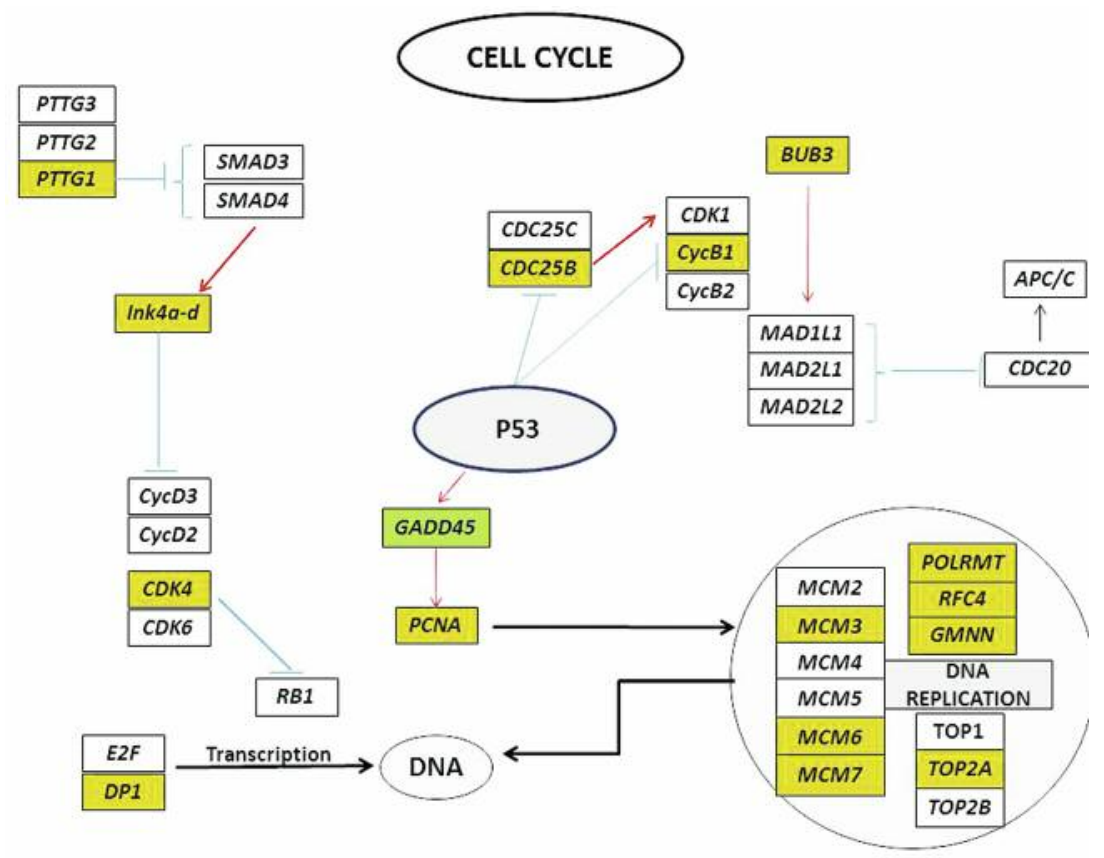

Figure 2. Dysregulated genes (fold change $\geq 2$ in $\geq 60 \%$ ) coding for cell-cycle regulation and DNA replication pictured using GenMAPPC software. Orange: Up-regulated genes; green: down-regulated genes. Red lines: activating effects; blue line: inhibiting effects. PTTG1-3: Pituitary transformingfactor 1-3; SMAD 3/4: SMAD family protein 3/4 (intracellular downstream proteins of transforming growth beta); Ink4a-d: cyclin dependent kinase inhibitor 2A; CycD2/3 CycB1/2: cyclin D2, D3, B1, B2; CDK1/4/6: cyclin dependent kinase 1, 4, 6; CDC25B/C: cell division cycle 25 B/C; CDC20: cell division cycle 20; APC: adenomatosis polyposis coli, WNT signaling pathway regulator; p53: p53 protein; GADD45: growth arrest and DNAdamage-inducible 45 alpha; PCNA: proliferating cell nuclear antigen; BUB3: mitotic checkpoint protein; MAD1/2: mitotic arrest deficient like 1/2; E2F: transcription factor E2F; DP1: transcription factor Dp1; MCM1-7: minichromosome maintenance complex component 1-7; GMNN: geminin; RFC4: replication factor C subunit 4; POLRMT: RNA polymerase mitochondrial; TOP1/2: topoisomerase 1/2; RB1: RB transcriptional corepressor 1. 
Table II. The 63 most commonly up-regulated genes in hepatocellular carcinoma up-regulated by at least two-fold in $\geq 80 \%$ of cases.

\begin{tabular}{|c|c|c|c|c|c|}
\hline Category & $\begin{array}{l}\text { Genbank } \\
\text { ID }\end{array}$ & $\begin{array}{l}\text { Gene } \\
\text { name }\end{array}$ & Function/description & $\begin{array}{l}\text { Fold } \\
\text { change }\end{array}$ & $\begin{array}{c}\% \\
\text { Cases }\end{array}$ \\
\hline \multirow[t]{4}{*}{ Cell cycle regulation } & D89729 & XPO1 & Exportin 1 & 2 & 100 \\
\hline & $\mathrm{U} 28386$ & KPNA2 & Karyopherin alpha 2 , importin alpha 1 & 3.3 & 93 \\
\hline & M61764 & TUBG1 & Gamma-tubulin & 3.9 & 80 \\
\hline & AL524035 & $C D C 2$ & Cell division cycle $2, G_{1}$ to $S$ and $G_{2}$ to $M$ & 3.1 & 80 \\
\hline \multirow[t]{5}{*}{ DNA replication } & X62153 & МСМЗ & MCM3 minichromosome maintenance deficient 3 & 3.1 & 100 \\
\hline & J04088 & TOP2A & DNA topological change & 19.6 & 83 \\
\hline & D84557 & МСM6 & MCM6 minichromosome maintenance deficient 6 & 3.8 & 80 \\
\hline & D55716 & $M C M 7$ & MCM7 minichromosome maintenance deficient 7 & 3.9 & 80 \\
\hline & J04718 & PCNA & DNA replication regulation; proliferating cell nuclear antigen & 2.7 & 80 \\
\hline \multirow[t]{13}{*}{ Transcription } & M69148 & $M D K$ & Retinoic-acid inducible growth regulator & 24 & 100 \\
\hline & NM_002131 & $H M G Y$ & High mobility group protein isoforms $\mathrm{i}$ and $\mathrm{y}$ & 7.6 & 100 \\
\hline & AK026573.1 & $B R G 1$ & Human transcriptional activator & 3.3 & 100 \\
\hline & NM_006713 & RNApol c4 & RNA polymerase II transcription cofactor 4 & 3 & 100 \\
\hline & NM_001571 & IRF3 & Interferon regulatory factor 3 & 3.0 & 100 \\
\hline & BC000903 & $H M G 2$ & High mobility group protein & 2.5 & 83 \\
\hline & $\mathrm{X} 14850$ & $H 2 A . X$ & Histone H2A.X, DNA binding & 3.9 & 80 \\
\hline & AJ249841 & KIF 22 & Kinesin-like DNA binding protein & 3.7 & 80 \\
\hline & S76638 & NFKB2 & NF-kappa B light polypeptide gene enhancer & 3.5 & 80 \\
\hline & X66899 & EWS & EWS, Ewing sarcoma related, interferes with transcription initiation & 3.1 & 80 \\
\hline & L23959 & TFDP1 & Transcription factor Dp-1 & 2.4 & 80 \\
\hline & U74612 & FOXM1 & HNF3/fork head box M1 & 2.1 & 80 \\
\hline & D14678 & KIFC1 & Kinesin-related protein & 2.1 & 80 \\
\hline \multirow{4}{*}{$\begin{array}{l}\text { Small GTPase- } \\
\text { interacting protein }\end{array}$} & L13687 & ARL2 & ADP-ribosylation factor-like 2 ,GTP binding, ras superfamily & 2.7 & 100 \\
\hline & NM_131187 & $C S N K 2 B$ & Casein kinase II subunit beta, MAPK signaling & 2.6 & 100 \\
\hline & L33930 & $C D 24$ & CD 24 signal transducer, antagonizes IL-mediated signals & 5.8 & 80 \\
\hline & AU153848 & $R A C G A P 1$ & Rac GTPase activating protein 1 & 3.8 & 80 \\
\hline \multirow{2}{*}{ Apoptosis } & U90426 & DDX39 & Nuclear RNA helicase, DEAD box family member & 2.4 & 80 \\
\hline & U33286 & CSE1L & Chromosome segregation gene homolog CAS, apoptosis related & 3.0 & 80 \\
\hline \multirow{5}{*}{$\begin{array}{l}\text { Ribosomal protein } \\
\text { synthesis/RNA } \\
\text { processing }\end{array}$} & NM_003093 & SNRPC & Small nuclear ribonucleoprotein polypeptide $\mathrm{C}$ & 8.6 & 100 \\
\hline & M60784 & SNRPA & Small nuclear ribonucleoprotein polypeptide A & 4.2 & 80 \\
\hline & L05096 & RPL39L & 60S ribosomal protein L39 & 3.9 & 80 \\
\hline & L03411.1 & $R D B P$ & RD RNA-binding protein & 3.2 & 80 \\
\hline & U15009 & SNRPD3 & Small nuclear ribonucleoprotein D3 polypeptide $18 \mathrm{kDa}$ & 2 & 80 \\
\hline \multirow{5}{*}{$\begin{array}{l}\text { Proteasome } \\
\text { degradation }\end{array}$} & M37583 & $H 2 A F Z$ & Histone $\mathrm{H} 2 \mathrm{~A}$ family, member $\mathrm{Z}$ & 2.2 & 80 \\
\hline & D44466 & PSMD1 & $26 \mathrm{~S}$ proteasome regulatory subunit $\mathrm{S} 1$ & 2.5 & 80 \\
\hline & NM 014501 & $E 2-E P F$ & Ubiqutin carrier protein & 2.9 & 80 \\
\hline & $\mathrm{U} 67 \overline{1} 22$ & $U B L$ & Ubiqutin-related protein, associating with RAD51/RAD52 proteins & 2.2 & 80 \\
\hline & U18919 & POV2 & Nucleotide binding protein, proteasome activator, BRCA1-related & 2.6 & 80 \\
\hline \multirow[t]{3}{*}{ Stress-related } & NM_005969 & NAP1L4 & Nucleosome assembly protein 1 -like 4 , chaperone, proliferative & 3.0 & 100 \\
\hline & BE737030 & ССТ6А & Chaperonin TCP1, subunit $6 \mathrm{~A}$ & 2.5 & 100 \\
\hline & Z23090 & HSPB1 & Heat-shock $27 \mathrm{kDa}$ protein & 2.4 & 80 \\
\hline Cytoskeleton/ & NM_001747 & $C A P G$ & Capping protein (actin-filament), gelsolin family & 8.6 & 100 \\
\hline \multirow[t]{18}{*}{ ECM modelling } & AK025912 & COLAA2 & Collagen, type IV, alpha 2 & 6.3 & 100 \\
\hline & NM_003118 & SPARC & Osteonectin, glycoprotein, ECM remodelling and cell growth regulator & 5.8 & 100 \\
\hline & NM_003289 & TPM2 & Tropomyosin 2 beta & 3.8 & 100 \\
\hline & AL031737 & $H D L C 1$ & Cytoplasmic dynein light chain 1 & 2.2 & 100 \\
\hline & NM_001855 & $A R C N 1$ & Archain 1, protein transporter & 2 & 100 \\
\hline & Y00705 & SPINK1 & Serine protease inhibitor, Kazal type 1 & 5.7 & 90 \\
\hline & L47125 & GPC3 & Glypican 3, proteoglycan cell surface, cell growth regulator & 8.3 & 80 \\
\hline & M83248 & $S P P 1$ & Osteopontin, secreted phosphoprotein, ECM interaction & 6.2 & 80 \\
\hline & M35252 & $T M 4 S F 3$ & Transmembrane 4 superfamily member 3 , tumor-associated antigen & 4.7 & 80 \\
\hline & L23808 & MMP12 & Matrix metalloproteinase 12 (macrophage elastase) & 4.4 & 80 \\
\hline & L23808 & $H M E$ & Metalloproteinase & 4.4 & 80 \\
\hline & BC001388 & ANXA2 & Annexin A2, links membrane phospholipids to actin & 3.5 & 80 \\
\hline & U75272 & $P G C$ & Gastricsin, peptidase, protease & 3.2 & 80 \\
\hline & X82456 & LASP1 & Actin binding protein, overexpressed in breast camcer & 2.5 & 80 \\
\hline & Y00503 & KRT19 & Cytokeratin 19 & 2.2 & 80 \\
\hline & X13839 & VSMA & Vascular smooth muscle alpha-actin & 2.1 & 80 \\
\hline & NM_001845 & COL4A1 & Collagen, type IV, alpha 1 & 2.1 & 80 \\
\hline & $\mathrm{Z} 74 \overline{6} 15$ & COL1A1 & Collagen, type I, alpha 1 & 2.1 & 80 \\
\hline \multirow[t]{2}{*}{ Tumor-associated } & AB018580 & $A K R 1 C 3$ & ALDO-ketoreductase family 1 member $\mathrm{C} 3$ & 2.9 & 87 \\
\hline & P32476 & ERG1 & Squalene monooxygenase & 2.7 & 87 \\
\hline \multirow[t]{2}{*}{ Miscellaneous } & BC001886 & RIR2 & Ribonucleotide reductase M2 polypeptide & 3 & 83 \\
\hline & AB016435 & EWSRI & RNA-binding protein EWS (EWS oncogene) & 3.1 & 80 \\
\hline
\end{tabular}


Table III. The 20 most significantly up-regulated genes in poorly differentiated (G3) hepatocellular carcinoma in comparison with well-differentiated tumors (G1).

\begin{tabular}{|c|c|c|}
\hline Affymetrix ID & $p$-Value & Gene description \\
\hline 203535_at & $7.56 \mathrm{E}+11$ & Homo sapiens $\mathrm{S} 100$ calcium-binding protein A9 (calgranulin B) (S100A9), mRNA \\
\hline 202917_s_at & $5.98 \mathrm{E}+11$ & H. sapiens S100 calcium-binding protein A8 (calgranulin A) (S100A8), mRNA \\
\hline 220510_at & $4.40 \mathrm{E}+11$ & H. sapiens $\mathrm{Rh}$ type B glycoprotein $(R H B G)$, mRNA \\
\hline 206894_at & $3.45 \mathrm{E}+11$ & H. sapiens apolipoprotein A-IV (APOA4), mRNA \\
\hline 204694_at & $2.96 \mathrm{E}+11$ & H. sapiens alpha-fetoprotein $(A F P)$, mRNA \\
\hline 204351_at & $2.57 \mathrm{E}+12$ & H. sapiens $\mathrm{S} 100$ calcium-binding protein $\mathrm{P}(\mathrm{S} 100 \mathrm{P})$, mRNA \\
\hline 212187_x_at & $1.53 \mathrm{E}+11$ & H. sapiens prostaglandin $\mathrm{D} 2$ synthase $(21 \mathrm{kDa}$, brain) $(P T G D S)$, mRNA \\
\hline 209752_at & $5.50 \mathrm{E}+10$ & H. sapiens lithostathine (REG1A) mRNA, complete cds \\
\hline 213479_at & $3.81 \mathrm{E}+11$ & Human neuronal pentraxin II (NPTX2) mRNA, partial cds \\
\hline 205815_at & $5.77 \mathrm{E}+09$ & H. sapiens pancreatitis-associated protein $(P A P)$, mRNA \\
\hline 207663_x_at & $2.56 \mathrm{E}+09$ & H. sapiens $\mathrm{G}$ antigen 3 (GAGE3), mRNA \\
\hline 208155_x_at & $4.50 \mathrm{E}+08$ & H. sapiens $\mathrm{G}$ antigen 6 (GAGEO), mRNA \\
\hline 207086_x_at & $3.62 \mathrm{E}+09$ & H. sapiens $\mathrm{G}$ antigen 4 (GAGE4), mRNA \\
\hline 208235_x_at & $1.30 \mathrm{E}+09$ & H. sapiens $\mathrm{G}$ antigen 7 (GAGE7), mRNA \\
\hline 207739_s_at & $5.25 \mathrm{E}+07$ & H. sapiens $\mathrm{G}$ antigen 2 (GAGE2), mRNA \\
\hline 206640_x_at & $5.25 \mathrm{E}+07$ & H. sapiens $\mathrm{G}$ antigen $7 \mathrm{~B}(G A G E 7 B)$, mRNA \\
\hline 201497_x_at & 0.00258 & H. sapiens myosin, heavy polypeptide 11 , smooth muscle (MYH11), mRNA \\
\hline 206291_at & 0.00258 & H. sapiens neurotensin $(N T S)$, mRNA \\
\hline 201839_s_at & 0.00295 & H. sapiens tumor-associated calcium signal transducer 1 (TACSTD1), mRNA \\
\hline 202768_at & 0.00327 & H. sapiens $\mathrm{FBJ}$ murine osteosarcoma viral oncogene homolog B $(F O S B)$, mRNA \\
\hline
\end{tabular}

Validation of gene-expression data by RT-PCR analysis. To validate the gene-expression results, the expression levels of four highly overexpressed genes (KPNA2, GPC3, SPINK1, $E R G 1)$ were evaluated by quantitative RT-PCR using the LightCycler system both in tumor and corresponding nonmalignant tissues. In general, the observed transcript levels in the microarray experiments correlated well with transcript levels from PCR light-cycler analysis. However, as has been shown previously $(23,24)$, the dynamic range of the realtime PCR results was typically about 1.5 - to 20 -fold higher when compared to that for the microarray data.

\section{Discussion}

One approach to understand how genetic and molecular changes are involved in cancer development is to examine abnormal gene expression in human tumors. To compare differentially expressed genes in human HCC tissue samples, we used oligonucleotide arrays, which have already been used successfully to identify novel genes in human hepatocarcinogenesis $(19,21)$. However, in previous reports, the HCC samples analyzed mostly originated from one single underlying form of liver disease (e.g. chronic hepatitis B or $\mathrm{C})$, thus creating a strong bias related to the different etiology of HCC or respective individual genetic background.

In this study, a specific gene-expression profile of 1,085 dysregulated genes (450 up- and 635 down-regulated genes) for $\mathrm{HCC}$ of different etiologies was generated based on an arbitrary relatively conservatively chosen algorithm. This profile was validated based on a neuronal training method by its ability to differentiate HCC profile from other expression profiles of corresponding and non-corresponding non-malignant liver tissues and other primary liver tumors (CCC). This method achieved not only a fast and reproducible differentiation between non-malignant disease and $\mathrm{HCC}$, but also a reliable differentiation between primary liver tumors (HCC vs. CCC) in general.

In contrast to $\mathrm{HCV}$-positive $\mathrm{HCC}$, which shows a more homogeneous genetic expression pattern, non-HCV-induced HCC samples were characterized by a far more heterogeneous distribution of genes, which is not surprising in view of the relatively wider range of different causes, e.g. alcoholic liver disease, NASH, and HBV infection leading to an accidental incorporation of viral DNA into the host genome.

By incorporating the expression data into GenMAPP software, a specific pattern of signaling and metabolic pathways, as well as changes in cell-cycle regulators specific for HCC, were generated. Generally, the changes in cell-cycle metabolism and a significant up-regulation of genes involved in DNA replication were well in line with a pattern of increased cell growth and underline the importance of uncontrolled cellcycle progression for human hepatocarcinogenesis.

Most of the other overexpressed genes in HCC belong to the group of genes important for extracellular matrix remodeling and regulation. In particular, the genes coding for the SPINK1 and GPC3 were recently reported to be 
dysregulated in HCC and are actually under investigation as potential screening markers for HCC or therapeutic targets (25-27). As described earlier by our group (28) SPINK1, also known as tumor-associated trypsin inhibitor, was typically overexpressed in HCV-positive HCCs, underlining the theory that SPINK1 may be able to interrupt apoptosis of viralinfected hepatocytes by cellular host defense (29) as one of the postulated mechanisms of this gene in hepatocarcinogegesis. Nevertheless, overexpression of SPINK1 was also seen in other types of human cancer, supporting the theory of SPINK1 being important factor for tumor invasiveness and metastasis (30).

Previous studies and our own data have shown a correlation between differentially expressed genes and tumor progression or tumor dedifferentiation in primary liver cancer $(23,31)$. In another analysis, we used twodimensional cluster analysis with all probe sets of the oligonucleotide array (>22,000 genes/ESTs) for the detection of differentially expressed genes in relation to tumor differentiation (G1 vs. G3 tumors). Not surprisingly, given the genetic heterogeneity and the small group of G1 tumors $(n=2)$, it was not possible to create an unambiguous clear gene-expression profile using all dysregulated genes and the histopathological grading. Nevertheless, 186 significantly dysregulated genes $(p<0.05)$ for potential differentiation between well- and poorly graded tumors were detected. In poorly differentiated (G3) tumors, G antigens such as GAGE2, -3, -4, -6, -7B and B1, melanoma antigens (MAGE family) and calcium-binding proteins (e.g. S100A9, S100A11 and S100P) were overexpressed. An overexpression of calcium-binging proteins such as S100A9 or S100A11 $(32,33)$ was described as important markers for tumor aggressiveness in poorly differentiated HCC and may be useful as markers for tumor prognosis or differentiation.

Similar observations have been reported for genes normally overexpressed in fetal liver such as AFP and neurotensin $(34,35)$. From our data, these genes were overexpressed, especially in poorly differentiated tumors, perhaps leading to a more aggressive behavior of these tumors (36).

In summary, using gene-expression analysis, a unique global genetic profile of $\mathrm{HCC}$, independently from the underlying chronic liver disease, was established and important involved molecular pathways were described. In relation to tumor differentiation, dysregulated genes were described with potential importance as prognostic markers and for a better understanding of tumor progression in human hepatocarcinogenesis.

\section{Conflicts of Interest}

The Authors declare no conflict of interest in regard to this study.

\section{Acknowledgements}

The Authors thank Stephan Kaiser (MD) for his support by the study. The study was supported by the fortüne-program of the University of Tübingen, No. F1281305.

\section{References}

1 World Health Organization. Mortality database. 2010 [updated 2010 February]; Available from: www.who.int/whosis/en.

2 Ortiz-Cuaran S, Villar S, Gouas D, Ferro G, Plymoth A, Khuhaprema T, Kalalak A, Sangrajrang S, Friesen MD, Groopman JD and Hainaut P: Association between HBX status, aflatoxin-induced R249S TP53 mutation and risk of hepatocellular carcinoma in a case-control study from Thailand. Cancer Lett 331: 46-51, 2013.

3 Qi LN, Bai T, Chen ZS, Qi LN, Bai T and Chen ZS: The $p 53$ mutation spectrum in hepatocellular carcinoma from Guangxi, China: role of chronic hepatitis B virus infection and aflatoxin B1 exposure. Liver Int 35: 35:999-1009, 2015.

4 Donato F, Tagger A, Chiesa R, Ribero ML, Tomasoni V, Fasola M, Gelatti U, Portera G, Boffetta P and Nardi G: Hepatitis B and $\mathrm{C}$ virus infection, alcohol drinking, and hepatocellular carcinoma: a case-control study in Italy. Brescia HCC Study. Hepatology 26: 579-84, 1997.

5 Gomaa AI, Khan SA, Toledano MB, Waked I and Taylor-Robinson SD: Hepatocellular carcinoma: epidemiology, risk factors and pathogenesis. World J Gastroenterol 14: 4300-4308, 2008.

6 Coulouarn C and Clément B: Stellate cells and the development of liver cancer: Therapeutic potential of targeting the stroma. J Hepatol 60: 1306-9, 2014.

7 Zhang X, Li HM, Liu Z, Zhou G, Zhang Q, Zhang T, Zhang J and Zhang C: Loss of heterozygosity and methylation of multiple tumor suppressor genes on chromosome 3 in hepatocellular carcinoma. J Gastroenterol 48: 132-143, 2013.

8 Xue W, Kitzing T, Roessler S, Zuber J, Krasnitz A, Schultz N, Revill K, Weissmueller S, Rappaport AR, Simon J, Zhang J, Luo W, Hicks J, Zender L, Wang XW, Powers S, Wigler M and Lowe SW: A cluster of cooperating tumor-suppressor gene candidates in chromosomal deletions. Proc Natl Acad Sci USA 109: 82128217,2012

9 Roessler S, Long EL, Budhu A, Chen Y, Zhao X, Ji J, Walker R, Jia HL, Ye QH, Qin LX, Tang ZY, He P, Hunter KW, Thorgeirsson SS, Meltzer PS and Wang XW: Integrative genomic identification of genes on $8 p$ associated with hepatocellular carcinoma progression and patient survival. Gastroenterology 142: 957-966, 2012.

10 Pannem RR, Dorn C, Ahlqvist K, Bosserhoff AK, Hellerbrand $\mathrm{C}$ and Massoumi R: CYLD controls c-MYC expression through the JNK-dependent signaling pathway in hepatocellular carcinoma. Carcinogenesis 35: 461-468, 2014.

11 Shiraha H, Yamamoto K and Namba M: Human hepatocyte carcinogenesis (review). Int J Oncol 42: 1133-1138, 2013.

12 Luo D, Liu QF, Gove C, Naomov N, Su JJ and Williams R: Analysis of N-RAS gene mutation and p53 gene expression in human hepatocellular carcinomas. World J Gastroenterol 4: 9799, 1998.

13 Reed CA, Mayhew CN, McClendon AK, Yang X, Witkiewicz A and Knudsen ES: RB has a critical role in mediating the in vivo 
checkpoint response, mitigating secondary DNA damage and suppressing liver tumorigenesis initiated by aflatoxin B1. Oncogene 28: 4434-4443, 2009.

14 Sørlie T, Perou CM, Tibshirani R, Aas T, Geisler S, Johnsen H, Hastie T, Eisen MB, van de Rijn M, Jeffrey SS, Thorsen T, Quist $\mathrm{H}$, Matese JC, Brown PO, Botstein D, Lønning PE and Børresen-Dale AL: Gene expression patterns of breast carcinomas distinguish tumor subclasses with clinical implications. Proc Natl Acad Sci USA 98: 10869-10874, 2001.

15 Calvo A, Xiao N, Kang J, Best CJ, Leiva I, Emmert-Buck MR, Jorcyk $\mathrm{C}$ and Green JE: Alterations in gene expression profiles during prostate cancer progression: functional correlations to tumorigenicity and down-regulation of selenoprotein-P in mouse and human tumors. Cancer Res 62: 5325-5335, 2002.

16 Udtha M, Lee SJ, Alam R, Coombes K and Huff V: Upregulation of $c-M Y C$ in WT1-mutant tumors: assessment of WT1 putative transcriptional targets using cDNA microarray expression profiling of genetically defined Wilms' tumors. Oncogene 22: 3821-3826, 2003.

17 Komori T, Takemasa I, Higuchi H, Yamasaki M, Ikeda M, Yamamoto H, Ohue M, Nakamori S, Sekimoto M, Matsubara K and Monden M: Identification of differentially expressed genes involved in colorectal carcinogenesis using a cDNA microarray. J Exp Clin Cancer Res 23: 521-527, 2004.

18 Ikehara M, Oshita F, Sekiyama A, Hamanaka N, Saito H, Yamada K, Noda K, Kameda Y and Miyagi Y: Genome-wide cDNA microarray screening to correlate gene expression profile with survival in patients with advanced lung cancer. Oncol Rep 11: 1041-1044, 2004

19 Iizuka N, Oka M, Yamada-Okabe H, Hamanaka N, Saito H, Yamada K, Noda K, Kameda Y and Miyagi Y: Molecular signature in three types of hepatocellular carcinoma with different viral origin by oligonucleotide microarray. Int J Oncol 24: 565-574, 2004.

20 Lee CF, Ling ZQ, Zhao T and Lee KR: Distinct expression patterns in hepatitis B virus- and hepatitis C virus-infected hepatocellular carcinoma. World J Gastroenterol 14: 6072-6077, 2008.

21 Lee SY, Song KH, Koo I, Lee KH, Suh KS and Kim BY: Comparison of pathways associated with hepatitis B- and Cinfected hepatocellular carcinoma using pathway-based class discrimination method. Genomics 99: 347-354, 2012.

22 Tilton SC, Gerwick LG, Hendricks JD, Rosato CS, Corley-Smith G, Givan SA, Bailey GS, Bayne CJ and Williams DE: Use of a rainbow trout oligonucleotide microarray to determine transcriptional patterns in aflatoxin B1-induced hepatocellular carcinoma compared to adjacent liver. Toxicol Sci 88: 319-330, 2005.

23 Hass HG, Nehls O, Jobst J, Frilling A, Vogel U and Kaiser S: Identification of osteopontin as the most consistently overexpressed gene in intrahepatic cholangiocarcinoma: detection by oligonucleotide microarray and real-time PCR analysis. World J Gastroenterol 14: 2501-2510, 2008.

24 Rajeevan MS, Vernon SD, Taysavang $\mathrm{N}$ and Unger ER: Validation of array-based gene expression profiles by real-time (kinetic) RT-PCR. J Mol Diagn 3: 26-31, 2001.
25 Holah NS, El-Azab DS, Aiad HA and Sweed DM: The diagnostic role of SPINK1 in differentiating hepatocellular carcinoma from nonmalignant lesions. Appl Immunohistochem Mol Morphol 2016. PMID:27028242. DOI:10.1097 /PAI.0000000000000363.

26 Hass HG, Jobst J, Scheurlen M, Vogel U and Nehls O: Gene expression analysis for evaluation of potential biomarkers in hepatocellular carcinoma. Anticancer Res 35: 2021-2028, 2015.

27 Filmus J and Capurro M: Glypican-3: a marker and a therapeutic target in hepatocellular carcinoma. FEBS J 280: 2471-2476, 2013.

28 Hass HG, Jobst J, Vogel U, Scheurlen M and Nehls O: Overexpression of tumor-associated trypsin inhibitor (SPINK1/TATI) in hepatitis C-associated hepatocellular carcinoma: potential implications for viral hepatocarcinogenesis. Oncol Res Treat 37: 732-738, 2014.

29 Lamontagne J, Pinkerton M, Block TM and Lu X: Hepatitis B and hepatitis $\mathrm{C}$ virus replication upregulates serine protease inhibitor Kazal, resulting in cellular resistance to serine proteasedependent apoptosis. J Virol 84: 907-917, 2010.

30 Flavin R, Pettersson A, Hendrickson WK, Fiorentino M, Finn S, Kunz L, Judson GL, Lis R, Bailey D, Fiore C, Nuttall E, Martin NE, Stack E, Penney KL, Rider JR, Sinnott J, Sweeney C, Sesso HD, Fall K, Giovannucci E, Kantoff P, Stampfer M, Loda M and Mucci LA: SPINK1 protein expression and prostate cancer progression. Clin Cancer Res 20: 4904-4911, 2014.

31 Damdinsuren B, Nagano H, Kondo M, Yamamoto H, Hiraoka N, Yamamoto T, Marubashi S, Miyamoto A, Umeshita K, Dono K, Nakamori S, Wakasa K, Sakon M and Monden M: Expression of Id proteins in human hepatocellular carcinoma: relevance to tumor dedifferentiation. Int J Oncol 26: 319-327, 2005.

$32 \mathrm{Wu}$ R, Duan L, Cui F, Cao J, Xiang Y, Tang Y and Zhou L: S100A9 promotes human hepatocellular carcinoma cell growth and invasion through RAGE-mediated ERK1/2 and p38 MAPK pathways. Exp Cell Res 334: 228-238, 2015.

33 Luo X, Xie H, Long X, Zhou M, Xu Z, Shi B, Jiang H and Li Z: EGFRvIII mediates hepatocellular carcinoma cell invasion by promoting S100 calcium binding protein A11 expression. PLoS One 8: e83332. doi: 10.1371/journal.pone.0083332, 2013.

34 Gambarin-Gelwan M, Wolf DC, Shapiro R, Schwartz ME and Min AD: Sensitivity of commonly available screening tests in detecting hepatocellular carcinoma in cirrhotic patients undergoing liver transplantation. Am J Gastroenterol 95: 15351538, 2000.

35 Ehrenfried JA, Zhou Z, Thompson JC and Evers BM: Expression of the neurotensin gene in fetal human liver and fibrolamellar carcinoma. Ann Surg 220: 484-489, 1994.

36 Zhao X, Parpart S, Takai A, Roessler S, Budhu A, Yu Z, Blank M, Zhang YE, Jia HL, Ye QH, Qin LX, Tang ZY, Thorgeirsson SS and Wang XW: Integrative genomics identifies YY1AP1 as an oncogenic driver in $\operatorname{EpCAM}(+) \mathrm{AFP}(+)$ hepatocellular carcinoma. Oncogene 34: 5095-5104, 2015.

Received August 5, 2016

Revised September 11, 2016

Accepted September 13, 2016 